\title{
Global weak conservative solutions of the $\mu$-Camassa-Holm equation
}

\author{
Xijun Deng ${ }^{1 *}$ and Aiyong Chen ${ }^{2}$
}

\section{"Correspondence:} xijundeng@aliyun.com

'Department of Mathematics and Physics, Hunan University of Arts and Science, Changde, China Full list of author information is available at the end of the article

\section{Springer}

\begin{abstract}
In this paper we consider global weak conservative solutions of a $\mu$-Camassa-Holm equation. By employing a technique of change of variables on the Lagrangian variable, we successfully construct global weak conservative solutions of the $\mu$-Camassa-Holm equation.
\end{abstract}

Keywords: Global weak conservative solutions; $\mu$-Camassa-Holm equation; Lagrangian variable

\section{Introduction}

In this paper, we are concerned with the Cauchy problem of the following periodic $\mu$ Camassa-Holm $(\mu \mathrm{CH})$ equation [1]:

$$
\left\{\begin{array}{l}
\mu\left(u_{t}\right)-u_{x x t}=-2 \mu(u) u_{x}+2 u_{x} u_{x x}+u u_{x x x}, \quad t>0, x \in S^{1}=\mathbb{R} / \mathbb{Z} \\
u(0, x)=u_{0}(x), \quad x \in S^{1}=\mathbb{R} / \mathbb{Z}
\end{array}\right.
$$

where $u(t, x)$ is a real-valued spatially periodic function and $\mu(u)=\int_{S^{1}} u(t, x) d x$ denotes its mean. This equation was introduced in [1] as an integrable equation arising in the study of the diffeomorphism group of the circle, and it can be viewed as a natural generalization of the famous Camassa-Holm $(\mathrm{CH})$ equation [2]

$$
u_{t}-u_{t x x}+3 u u_{x}-2 u_{x} u_{x x}-u u_{x x x}=0
$$

Moreover, $\mu \mathrm{CH}$ equation (1) has some properties similar to $\mathrm{CH}$ equation (2). For instance, like $\mathrm{CH}$ equation (2), $\mu \mathrm{CH}$ equation (1) is also completely integrable in the sense that it has a Lax pair representation, a bi-Hamiltonian structure, an infinite sequence of conservation laws, and peaked soliton solutions (called peakons). $\mu \mathrm{CH}$ equation (1) also arises geometrically as equations for geodesic flow in the context of the diffeomorphism group of the circle Diff $\left(S^{1}\right)$ endowed with a right-invariant Riemannian metric induced by the $\mu$ inner product $\langle u, v\rangle=\mu(u) \mu(v)+\int_{S^{1}} u_{x} v_{x} d x$.

Recently, well-posedness and wave breaking of the $\mathrm{CH}$ equation have been studied extensively. More precisely, it was shown in $[3,4]$ that the $\mathrm{CH}$ equation has a unique solution in $C\left([0, T), H^{s}(\mathbb{R})\right)$ with $s>\frac{3}{2}$. It was proved in [5] that the $\mathrm{CH}$ equation is locally well posed

(c) The Author(s) 2020. This article is licensed under a Creative Commons Attribution 4.0 International License, which permits use, sharing, adaptation, distribution and reproduction in any medium or format, as long as you give appropriate credit to the original author(s) and the source, provide a link to the Creative Commons licence, and indicate if changes were made. The images or other third party material in this article are included in the article's Creative Commons licence, unless indicated otherwise in a credit line to the material. If material is not included in the article's Creative Commons licence and your intended use is not permitted by statutory regulation or exceeds the permitted use, you will need to obtain permission directly from the copyright holder. To view a copy of this licence, visit http://creativecommons.org/licenses/by/4.0/. 
in Besov spaces $C\left([0, T), B_{p, r}^{s}(\mathbb{R})\right)$ with $s>\max \left(\frac{3}{2}, 1+\frac{1}{p}\right), 1 \leq p \leq+\infty, 1 \leq r<+\infty$. It was shown in [6-8] that the strong solutions of $\mathrm{CH}$ equation will blow up in finite time when the slope of initial data was bounded by a negative quantity. Also, well-posedness and wave breaking of the $\mu \mathrm{CH}$ equation were studied and some results about the local and global existence and uniqueness in Sobolev spaces were established in [1]. Moreover, the $\mu \mathrm{CH}$ equation admits a similar phenomenon as the $\mathrm{CH}$ equation, that is, the solutions break down in finite time when the initial data satisfy some proper conditions. In particular, it was shown in [9] that $\mu \mathrm{CH}$ equation (1) also admits peakons $u(x, t)=c \phi(x-c t)$, where $\phi(x)=\frac{1}{26}\left(12 x^{2}+23\right)$ for $x \in\left[-\frac{1}{2}, \frac{1}{2}\right]$, and $\phi$ is extended periodically to the real line. Chen, Lenells, and Liu [10] showed that the above periodic peakons are orbitally stable.

In order to study solutions of $\mathrm{CH}$ equation (2) after the time of break-down, Bressan and Constantin $[11,12]$ introduce some new variables to resolve the singularities formed by wave breaking, and they successfully obtain a semigroup of global solutions by returning to the original variables. Holden and Raynaud $[13,14]$ also study solutions of $\mathrm{CH}$ equation (2) after the formation of singularities, but they introduce a set of different new variables, and this can establish a bijective map between Eulerian and Lagrangian coordinates for $\mathrm{CH}$ equation (2). Motivated by the works of Lenells [15], who constructed global weak conservative solutions of the so-called periodic Hunter-Saxton (HS) equation

$$
u_{t x x}+2 u_{x} u_{x x}+u u_{x x x}=0
$$

Lee [16] constructs global weak conservative solutions of $\mathrm{CH}$ equation (2) by using transformation of variables on the Lagrangian variable $\eta$ :

$$
\rho=\sqrt{\eta_{x}}
$$

It is interesting that the author obtains the same global results for $\mathrm{CH}$ equation (2) as those presented by Bressan and Constantin [11] and also improves the corresponding results on the persistence of the smoothness of Lagrangian trajectories presented in [17]. More recently, following closely the ideas used in [12,14], Tiğlay [18] studies the periodic Cauchy problem for $\mu \mathrm{CH}$ equation (1) and proves the existence and uniqueness of conservative weak solutions.

In this paper, we investigate the global weak conservative solutions of $\mu \mathrm{CH}$ equation (1). We closely follow the main ideas of Lee [16] in the study of conservative weak solutions of $\mathrm{CH}$ equation (2). We show that the wave breaking singularities of $\mu \mathrm{CH}$ equation (1) can be removed by introducing the same transformation (4) about the new Lagrangian variable $\rho$, even though the structure of the $\mu \mathrm{CH}$ equation is in some sense different from the $\mathrm{CH}$ equation and the HS equation. Also, we obtain the global results of the $\mu \mathrm{CH}$ equation due to Tiğlay [18] in much simpler way.

The paper is organized as follows. In Sect. 2, we use the Lagrangian variable $\rho$ defined by (4) to change $\mu \mathrm{CH}$ equation (1) into an abstract ODE system which admits global solutions. In Sect. 3, we construct global weak conservative solutions of the original $\mu \mathrm{CH}$ equation by using this global solution in $\rho$ variables. Moreover, by putting together all the previous results obtained, we give and prove the main result of the paper. Finally, some conclusions and discussions are presented in Sect. 4. 


\section{The setting}

Firstly, the Cauchy problem for periodic $\mu \mathrm{CH}$ equation (1) can be rewritten in the weak form

$$
\left\{\begin{array}{l}
u_{t}+u u_{x}=-\left(\mu-\partial_{x}^{2}\right)^{-1} \partial_{x}\left(2 \mu(u) u+\frac{1}{2} u_{x}^{2}\right) \\
u(0, x)=u_{0}(x)
\end{array}\right.
$$

By taking methods analogous to those in [16], we define the following flow equation in terms of Lagrangian variable $\eta$ :

$$
\frac{\partial \eta}{\partial t}=u(t, \eta(t, x))
$$

then Cauchy problem (5) can be written in $\eta$ variable as follows:

$$
\left\{\begin{array}{l}
\eta_{t t}=-\left[\Lambda^{-1} \partial_{x}\left(2 \mu \eta_{t} \circ \eta^{-1}+\frac{1}{2}\left(\frac{\eta_{t x}}{\eta_{x}} \circ \eta^{-1}\right)^{2}\right)\right] \circ \eta \\
\eta(0, x)=x, \quad \eta_{t}(0, x)=u_{0}(x)
\end{array}\right.
$$

where $\Lambda^{-1}=\left(\mu-\partial_{x}^{2}\right)^{-1}$ is the operator defined by

$$
\Lambda^{-1} u(x)=\int_{S^{1}} g(x-y) u(y) d y,
$$

and the kernel $g(x)$ of the elliptic operator $\Lambda^{-1}$ is given by $g(x)=\frac{1}{2}\left(x^{2}-|x|\right)+\frac{13}{12}$.

Now our main aim is to write Eq. (7) in terms of the new variable $\rho$ defined by (4). For this purpose, we take full advantage of the conserved quantities $\mu(u)$ of the $\mu \mathrm{CH}$ equation to finish our work. More precisely, note that $\mu(u)=\int_{0}^{1} u(t, x) d x=\int_{0}^{1} u_{0}(x) d x$ is a constant determined by the initial condition $u_{0}$, so in view of (4) and (6) we have

$$
\mu=\int_{0}^{1}(u \circ \eta) \cdot \eta_{x} d x=\int_{0}^{1} \eta_{t} \eta_{x} d x=\int_{0}^{1} \eta_{t} \rho^{2} d x
$$

By carrying out exactly similar arguments as those given in [16], we can determine $\eta_{t}$ in terms of $\mu, \rho$, and $\rho_{t}$. That is,

$$
\eta_{t}:=G\left(\mu, \rho, \rho_{t}\right)(t, x)=\int_{0}^{x} 2 \rho \rho_{t} d y+\mu-\int_{0}^{1} \int_{0}^{y} 2 \rho \rho_{t} d z \rho^{2} d y .
$$

Also, notice that $\rho=\sqrt{\eta_{x}}$ and $\eta_{t x}=2 \rho \rho_{t}$, then we have $\frac{\eta_{t x}^{2}}{2 \eta_{x}}=2 \rho_{t}^{2}$.

Next, we begin to rewrite Eq. (7) in $\rho$ variables. By differentiating Eq. (7) with respect to $x$, we have

$$
\eta_{t t x}=2 \mu \eta_{t} \eta_{x}+\frac{\eta_{t x}^{2}}{2 \eta_{x}}-\left[\int_{0}^{1}\left(2 \mu \eta_{t} \eta_{y}+\frac{\eta_{t y}^{2}}{2 \eta_{y}}\right) d y\right] \cdot \eta_{x}
$$

where we have applied the chain rule and the identity $\Lambda^{-1}\left(-\partial_{x}^{2}\right)=1-\mu$. For convenience, we denote that

$$
F\left(\mu, \rho, \rho_{t}\right)(t):=\int_{0}^{1} 2 \mu \rho^{2} G+2 \rho_{t}^{2} d x
$$


so Eq. (10) becomes

$$
2 \rho \rho_{t t}=2 \mu G \rho^{2}-F \rho^{2}
$$

since $\eta_{t t x}-\frac{\eta_{t x}^{2}}{2 \eta_{x}}=2 \rho \rho_{t t}$. Thus, we can derive the following Cauchy problem with respect to $\rho$ variables:

$$
\left\{\begin{array}{l}
\rho_{t t}=\rho\left(\mu G-\frac{1}{2} F\right), \\
\rho(0, x)=1, \quad \rho_{t}(0, x)=\frac{1}{2} u_{0}^{\prime}(x) .
\end{array}\right.
$$

If we assume that $\rho$ and $\rho_{t}$ are just functions in $L^{2}\left(S^{1}\right)$ satisfying Eq. (13), then this equation can be used to describe the integral curve of the vector field $\left(\rho, \rho_{t}\right) \mapsto f\left(\rho, \rho_{t}\right):=$ $\rho\left(\mu G\left(\rho, \rho_{t}\right)-\frac{1}{2} F\left(\rho, \rho_{t}\right)\right)$. According to the smoothness of $f$ with respect to $\rho$ and $\rho_{t}$ variables, we can conclude that the solutions of (13) are locally well posed.

Proposition 2.1 The planar system

$$
\left\{\begin{array}{l}
\frac{d \rho}{d t}=\rho_{t} \\
\frac{d \rho_{t}}{d t}=f\left(\rho, \rho_{t}\right):=\rho\left(\mu G\left(\rho, \rho_{t}\right)-\frac{1}{2} F\left(\rho, \rho_{t}\right)\right)
\end{array}\right.
$$

with initial conditions $\rho(0, \cdot)=1$ and $\rho_{t}(0, \cdot)=\frac{1}{2} u_{0}^{\prime}$ describes the flow of a $C^{\infty}$ vector field on $T L^{2}\left(S^{1}\right)=L^{2}\left(S^{1}\right) \times L^{2}\left(S^{1}\right)$, and there exists $T>0$ such that it has a unique solution $\left(\rho, \rho_{t}\right) \in C\left([0, T] ; T L^{2}\left(S^{1}\right)\right)$.

Proof Firstly, note that

$$
\frac{\partial f}{\partial \rho_{t}}=\rho\left(\mu \frac{\partial G}{\partial \rho_{t}}-\frac{1}{2} \frac{\partial F}{\partial \rho_{t}}\right) .
$$

It is easy to see that $G$ is smooth with respect to $\rho_{t}$ since it is linear in $\rho_{t}$. On the other hand, we have

$$
\frac{\partial F}{\partial \rho_{t}}=\int_{0}^{1} 2 \mu \rho^{2} \frac{\partial G}{\partial \rho_{t}}+4 \rho_{t} d x,
$$

and so we can observe that $F$ is also smooth with respect to $\rho_{t}$. Thus, it implies that the vector field $f$ is smooth with respect to $\rho_{t}$. Similarly, we can check that the vector field $f$ is smooth with respect to $\rho$.

Note that $G$ is periodic on $S^{1}$ if and only if $\int_{0}^{1} \rho^{2} d x=0$. The following proposition shows that we need to restrict $\rho$ to be on the unit sphere $U:=\left\{\rho \in L^{2}\left(S^{1}\right):\|\rho\|_{L^{2}}=1\right\}$ if we want to obtain the global well-posedness of Cauchy problem (13).

Proposition 2.2 Let $f\left(\rho, \rho_{t}\right)$ be the vector field on $T L^{2}\left(S^{1}\right)$ defined by (13). If $\rho \in U$, then $\int_{0}^{1} \rho \rho_{t} d x=0$ for all time. This implies that $f$ restricts on the tangent bundle TU.

Proof When $t=0$, we have $\int_{0}^{1} \rho(0, x) \rho_{t}(0, x)=\int_{0}^{1} \frac{1}{2} u_{0}^{\prime}(x) d x=\frac{1}{2}\left(u_{0}(1)-u_{0}(0)\right)=0$. Note that $F$ does not depend on $x$ and the assumption that $\rho \in U$, so we have $\int_{0}^{1} \rho^{2} F d x=F$. 
Thus, in view of (11), we have

$$
\frac{d}{d t} \int_{0}^{1} \rho \rho_{t} d x=\int_{0}^{1} \rho_{t}^{2}+\rho \rho_{t t} d x=\int_{0}^{1} \rho_{t}^{2}+\rho^{2}\left(\mu G-\frac{1}{2} F\right) d x=\frac{1}{2} F-\frac{1}{2} F=0
$$

This proves that $\int_{0}^{1} \rho \rho_{t} d x=0$ for all time since the integral is initially zero.

It is well known that the $\dot{H}^{1}$ energy $\int_{0}^{1} u_{x}^{2} d x$ is a conserved quantity for $\mu \mathrm{CH}$ equation (1). Next, we show that the $\dot{H}^{1}$ energy for the corresponding $\rho$ equation is also conserved.

Proposition 2.3 For the $\rho$ equation, we have

$$
\frac{d}{d t} \int_{0}^{1} 4 \rho_{t}^{2} d x=0
$$

Proof Recall that

$$
G_{x}=2 \rho \rho_{t} .
$$

Then we have

$$
\begin{aligned}
\frac{d}{d t} \int_{0}^{1} 4 \rho_{t}^{2} d x & =\int_{0}^{1} 8 \rho_{t} \rho_{t t} d x \\
& =\int_{0}^{1} 4 \rho \rho_{t}(2 \mu G-F) d x \\
& =2 \mu \int_{0}^{1} 2 G G_{x} d x-2 F \int_{0}^{1} 2 \rho \rho_{t} d x \\
& =2 \mu \int_{0}^{1} d\left(G^{2}\right)-2 F \int_{0}^{1} d(G)=0
\end{aligned}
$$

since $G$ is periodic with respect to $x$.

Now, we can show that the solution of Eq. (13) is global when $\rho$ is restricted on the unit sphere.

Proposition 2.4 If $\rho \in U$, then the flow described by (13) exists for all time $t$.

Proof In view of Hölder's inequality, we have

$$
|2 \mu G-F| \leq 2 \mu|G|+|F| \leq 4 \mu\|\rho\|_{L^{2}}\left\|\rho_{t}\right\|_{L^{2}}+4 \mu\|\rho\|_{L^{2}}^{3}\left\|\rho_{t}\right\|_{L^{2}}+2\left\|\rho_{t}\right\|_{L^{2}}^{2}
$$

It is easily seen that $|2 \mu G-F|$ is uniformly bounded since $\|\rho\|_{L^{2}}=1$ and $\left\|\rho_{t}\right\|_{L^{2}}$ is uniformly bounded by the energy conservation. This implies that the right-hand side of (13) is uniformly bounded in $\left(\rho, \rho_{t}\right)$. Thus, by Wintner's theorem from the ODE theory [19], we can conclude that the solutions of Cauchy problem (13) exist for all time. 


\section{Global weak solutions of the $\mu \mathrm{CH}$ equation}

We want to construct global weak solutions for the original $\mu \mathrm{CH}$ equation (5) from the global solution $\rho$. To do this, we first prove the following important lemma.

Lemma 3.1 Let $G$ be defined by (9). Then $G_{t}=-H$, where

$$
\begin{aligned}
H(t, x)= & \int_{0}^{1}\left(\int_{y}^{x} \rho^{2} d z\right)\left(2 \mu G \rho^{2}+2 \rho_{t}^{2}\right) d y \\
& +\frac{1}{2} \int_{x}^{1} 2 \mu \rho^{2} G+2 \rho_{t}^{2} d y-\frac{1}{2} \int_{0}^{x} 2 \mu \rho^{2} G+2 \rho_{t}^{2} d y .
\end{aligned}
$$

Proof Note that

$$
\frac{\partial H}{\partial x}=\rho^{2} F-\left(2 \mu \rho^{2} G+2 \rho_{t}^{2}\right)
$$

and

$$
H \rho^{2}=\Phi_{x},
$$

where

$$
\Phi(t, x)=\int_{0}^{1}\left[\left(\int_{y}^{x} \rho^{2} d z\right)^{2}-\left|\int_{y}^{x} \rho^{2} d z\right|\right]\left(\mu \rho^{2} G+\rho_{t}^{2}\right) d y .
$$

Denote

$$
c(t):=\mu-\int_{0}^{1} \int_{0}^{x} 2 \rho \rho_{t} d y \rho^{2} d x .
$$

By differentiating $G$ with respect to $t$, we have

$$
\begin{aligned}
G_{t} & =\int_{0}^{x} 2 \rho_{t}^{2}+2 \rho \rho_{t t} d y+c^{\prime}(t) \\
& =\int_{0}^{x} 2 \rho_{t}^{2}+2 \mu \rho^{2} G-\rho^{2} F d y+c^{\prime}(t) \\
& =-\int_{0}^{x} \frac{\partial H}{\partial y} d y+c^{\prime}(t) \\
& =-H(t, x)+H(t, 0)+c^{\prime}(t) .
\end{aligned}
$$

So we only need to show that $H(t, 0)+c^{\prime}(t)=0$. However, we have

$$
\begin{aligned}
c^{\prime}(t) & =-\int_{0}^{1} \int_{0}^{x} 2 \rho_{t}^{2}+2 \rho \rho_{t t} d y \rho^{2} d x \\
& =-\int_{0}^{1} \int_{0}^{x} 2 \rho_{t}^{2}+2 \mu \rho^{2} G-\rho^{2} F d y \rho^{2} d x \\
& =-\int_{0}^{1}\left(2 \rho_{t}^{2}+2 \mu \rho^{2} G-\rho^{2} F\right) \int_{y}^{1} \rho^{2} d x d y
\end{aligned}
$$




$$
\begin{aligned}
& =\int_{0}^{1} \frac{\partial H}{\partial y} \int_{y}^{1} \rho^{2} d x d y \\
& =\int_{0}^{1}\left[\rho^{2} \int_{0}^{x} \frac{\partial H}{\partial y} d y\right] d x \\
& =\int_{0}^{1}\left[\rho^{2} H(t, x)-\rho^{2} H(t, 0)\right] d x \\
& =\int_{0}^{1} \Phi_{x} d x-H(t, 0) \int_{0}^{1} \rho^{2} d x \\
& =-H(t, 0),
\end{aligned}
$$

since $\int_{0}^{1} \rho^{2} d x=1$ and $\Phi$ is periodic with respect to $x$. Therefore, we have $G_{t}=-H$.

Define

$$
K(t, x):=\int_{0}^{x} \rho^{2} d y+t u_{0}(0)-\int_{0}^{t} \int_{0}^{\tau} H(s, 0) d s d \tau .
$$

From Lemma 3.1, we can see that $-H(t, 0)=c^{\prime}(t)$, where $c(t)$ is defined by (17). So the relation $\frac{\partial K}{\partial t}=G$ holds true between $K$ and $G$. Next, we will show that $(K, G)$ satisfies the second order equation (7).

Following $[15,16]$, we decompose $S^{1}$ by $S^{1}=N \cup A \cup Z$ where

$$
\begin{aligned}
& N:=\left\{x \in S^{1}: K_{x}=0 \text {, i.e., } \rho(t, x)=0\right\} \\
& A:=\left\{x \in S^{1}: K_{x}>0 \text {, i.e., } \rho(t, x)>0\right\}
\end{aligned}
$$

and meas $(Z)=0$. Performing the same argument as in [16], we can obtain that the set $N$ has a measure zero for almost all time. And then we have the following lemma.

Lemma 3.2 $\int_{N} \rho_{t}^{2} d y=0$ for almost all time $t \in \mathbb{R}_{+}$.

Proof The proof is very similar to that of Lemma 8 in [16], and hence is omitted.

Lemma 3.2 shows that $\rho=0$ is satisfied only on a set of measure zero for almost all time. Notice that $K_{x}=\rho^{2}$, we can derive that $K$ is generically a homeomorphism except for a set of measure zero. In other words, $K^{-1}$ is well defined for almost all time.

Proposition 3.3 For almost all time $t,(K, G)$ satisfies the following equation:

$$
G_{t}=-\left[\Lambda^{-1} \partial_{x}\left(2 \mu G \circ K^{-1}+\frac{1}{2}\left(\frac{G_{x}}{K_{x}} \circ K^{-1}\right)^{2}\right)\right] \circ K
$$

Proof It suffices to show that $G_{t}=-H$ is equivalent to Eq. (7) in the weak sense. Note that $K$ is a diffeomorphism on the set $A$, so we have

$$
\begin{aligned}
G_{t} & =-H=-\int_{S^{1}}\left[\int_{y}^{x} \rho^{2} d z-\frac{1}{2} \operatorname{sgn}\left(\int_{y}^{x} \rho^{2} d z\right)\right]\left(2 \mu \rho^{2} G+2 \rho_{t}^{2}\right) d y \\
& =-\int_{A}\left[K(x)-K(y)-\frac{1}{2} \operatorname{sgn}(K(x)-K(y))\right]\left(2 \mu K_{x} G+\frac{G_{x}^{2}}{2 K_{x}}\right) d y+\int_{N} \rho_{t}^{2} d y
\end{aligned}
$$




$$
\begin{aligned}
= & -\int_{K(A)}\left[K(x)-y-\frac{1}{2} \operatorname{sgn}(K(x)-y)\right]\left(2 \mu G \circ K^{-1}+\frac{1}{2}\left(\frac{G_{x}}{K_{x}} \circ K^{-1}\right)^{2}\right) d y \\
& +\int_{N} \rho_{t}^{2} d y \\
= & -\partial_{x}\left[\int_{K(A)}\left(\frac{(x-y)^{2}}{2}-\frac{|x-y|}{2}+\frac{13}{12}\right)\left(2 \mu G \circ K^{-1}+\frac{1}{2}\left(\frac{G_{x}}{K_{x}} \circ K^{-1}\right)^{2}\right) d y\right] \circ K \\
& +\int_{N} \rho_{t}^{2} d y .
\end{aligned}
$$

In view of that meas $(K(A))=1$, we can observe that the first integral of the above is equivalent to the right-hand side of Eq. (7). Moreover, we have $\int_{N} \rho_{t}^{2} d y=0$ for almost all time $t$ from Lemma 3.2. Therefore, for almost all time $t,(K, G)$ satisfies Eq. (18).

Next, we check that the velocity field $u$ satisfying flow equation (6) is well defined in $H^{1}$ for all time. More precisely, we can obtain the following proposition. It should be remarked here that the proofs for this proposition are omitted since it is exactly the same argument as in [16].

Proposition 3.4 Let $(K, G)$ be a weak solution of $\mu C H$ equation (7) in the Lagrangian form. Then the velocity field $u \in C\left(\mathbb{R}_{+}, H^{1}\left(S^{1}\right)\right) \cap \operatorname{Lip}\left(\mathbb{R}_{+}, L^{2}\left(S^{1}\right)\right)$ is well defined by

$$
u(t, K(t, x))=G(t, x), \quad(t, x) \in[0, \infty) \times S^{1}
$$

Now, we are in a position to state the main result of this paper.

Theorem 3.5 The function $u$ defined by (19) is the global weak solution to the Cauchy problem for periodic $\mu \mathrm{CH}$ equation (5). Moreover, this solution satisfies the following property: $\int_{S^{1}} u_{x}^{2}(t, x) d x=\int_{S^{1}}\left(u_{0}^{\prime}(x)\right)^{2} d x$.

Proof We want to show that the function $u$ defined by (19) satisfies Eq. (5) in the weak sense. That is, it suffices to prove that

$$
\int_{S^{1} \times R_{+}}\left(u_{t}+u u_{x}\right) \varphi d x d t=\int_{S^{1} \times R_{+}}-p_{x} \varphi d x d t
$$

for all $\varphi \in C_{0}^{\infty}\left(S^{1} \times R_{+}\right)$, where $p=\Lambda^{-1}\left(2 \mu(u) u+\frac{1}{2} u_{x}^{2}\right)$. By virtue of (19), we have

$$
\begin{aligned}
\int_{S^{1} \times R_{+}}\left(u_{t}+u u_{x}\right) \varphi d x d t & =\int_{S^{1} \times R_{+}}-u \varphi_{t}+u u_{x} \varphi d x d t \\
& =\int_{S^{1} \times R_{+}}-G K_{x} \varphi_{t} \circ K+G G_{x} \varphi \circ K d x d t .
\end{aligned}
$$

Note that $K_{t}=G$ and $K_{t x}=G_{x}$, so we have

$$
\begin{aligned}
& \left(G K_{x} \varphi \circ K\right)_{t}-\left(G^{2} \varphi \circ K\right)_{x} \\
& =G_{t} K_{x} \varphi \circ K+G K_{t x} \varphi \circ K+G K_{x} \varphi_{t} \circ K+G K_{x}\left(\varphi_{x} \circ K\right) K_{t} \\
& \quad-2 G G_{x} \varphi \circ K-G^{2}\left(\varphi_{x} \circ K\right) K_{x}
\end{aligned}
$$




$$
\begin{aligned}
= & G_{t} K_{x} \varphi \circ K+G G_{x} \varphi \circ K+G K_{x} \varphi_{t} \circ K+G^{2} K_{x}\left(\varphi_{x} \circ K\right) \\
& -2 G G_{x} \varphi \circ K-G^{2}\left(\varphi_{x} \circ K\right) K_{x} \\
= & G_{t} K_{x} \varphi \circ K-G G_{x} \varphi \circ K+G K_{x} \varphi_{t} \circ K .
\end{aligned}
$$

Thus, in view of that $\varphi$ has a compact support, we can get

$$
\int_{S^{1} \times R_{+}}-G K_{x} \varphi_{t} \circ K+G G_{x} \varphi \circ K d x d t=\int_{S^{1} \times R_{+}} G_{t} K_{x} \varphi \circ K d x d t
$$

On the other hand, from Proposition 3.3 we know that $G_{t}=-\left[\Lambda^{-1} \partial_{x}\left(2 \mu G \circ K^{-1}\right.\right.$ $\left.\left.+\frac{1}{2}\left(\frac{G_{x}}{K_{x}} \circ K^{-1}\right)^{2}\right)\right] \circ K$ for almost all $t$. Also, note that $u=G \circ K^{-1}$ and $u_{x}=\frac{G_{x}}{K_{x}} \circ K^{-1}$. Therefore, we have

$$
\int_{S^{1} \times R_{+}} G_{t} K_{x} \varphi \circ K d x d t=\int_{S^{1} \times R_{+}}-p_{x} \varphi d x d t
$$

So we complete the proof of (20).

Next, we show that $\int_{S^{1}} u_{x}^{2}(t, x) d x$ is a conserved quantity for $\mu \mathrm{CH}$ equation (5). Note that $K_{x}=\rho^{2}$ and $G_{x}=2 \rho \rho_{t}$, then

$$
\begin{aligned}
\int_{S^{1}} u_{x}^{2} d x & =\int_{K(A)}\left(\frac{G_{x}}{K_{x}} \circ K^{-1}\right)^{2} d x \\
& =\int_{A} \frac{G_{x}^{2}}{K_{x}} d x \\
& =\int_{S^{1}} 4 \rho_{t}^{2} d x
\end{aligned}
$$

is conserved by virtue of Proposition 2.3. This proves that $\int_{S^{1}} u_{x}^{2}(t, x) d x=\int_{S^{1}}\left(u_{0}^{\prime}(x)\right)^{2} d x$.

\section{Conclusions}

In this paper, we successfully construct global weak conservative solutions of the $\mu \mathrm{CH}$ equation by applying the so-called Lagrangian change of variable technique. We believe that this technique could be extended to study other generalized $\mu$-type equations with different nonlocal nonlinearities which have similar remarkable properties as the $\mu \mathrm{CH}$ equation. More recently, by virtue of an isometric embedding of the group of diffeomorphism group, Gallouët and Vialard [20] have rewritten the $\mathrm{CH}$ equation as an incompressible Euler equation on the cone manifold. Following this geometric point of view developed in [20], we think that the $\mu \mathrm{CH}$ equation can be also embedded in the incompressible Euler equation on a non-compact Riemannian manifold. We leave these for future research. 
Availability of data and materials

Not applicable.

\section{Competing interests}

The authors declare that there are no competing interests.

\section{Authors' contributions}

Two authors cooperated to complete the work. All authors read and approved the final manuscript.

\section{Author details}

'Department of Mathematics and Physics, Hunan University of Arts and Science, Changde, China. ${ }^{2}$ Department of Mathematics, Hunan First Normal University, Changsha, China.

\section{Publisher's Note}

Springer Nature remains neutral with regard to jurisdictional claims in published maps and institutional affiliations.

Received: 14 October 2019 Accepted: 12 February 2020 Published online: 24 February 2020

\section{References}

1. Khesin, B., Lenells, J., Misiolek, G.: Generalized Hunter-Saxton equation and the geometry of the group of circle diffeomorphisms. Math. Ann. 342(3), 617-656 (2008)

2. Camassa, R., Holm, D.D.: An integrable shallow water equation with peaked solitons. Phys. Rev. Lett. 71(11), 1661-1664 (1993)

3. Constantin, A., Escher, J.: Well-posedness, global existence, and blowup phenomena for a periodic quasi-linear hyperbolic equation. Commun. Pure Appl. Math. 51(5), 475-504 (1998)

4. Rodriguez-Blanco, G.: On the Cauchy problem for the Camassa-Holm equation. Nonlinear Anal. 46(3), 309-327 (2001)

5. Danchin, R.: A few remarks on the Camassa-Holm equation. Differ. Integral Equ. 14(8), 953-988 (2001)

6. Constantin, A.: Existence of permanent and breaking waves for a shallow water equation: a geometric approach. Ann. Inst. Fourier (Grenoble) 50(2), 321-362 (2000)

7. Constantin, A., Escher, J.: Global existence and blow-up for a shallow water equation. Ann. Sc. Norm. Super. Pisa, Cl. Sci. (4) 26(2), 303-328 (1998)

8. Constantin, A., Escher, J.: Wave breaking for nonlinear nonlocal shallow water equations. Acta Math. 181(2), 229-243 (1998)

9. Lenells, J., Misiołek, G., Tiğlay, F.: Integrable evolution equations on spaces of tensor densities and their peakon solutions. Commun. Math. Phys. 299, 129-161 (2010)

10. Chen, M., Lenells, J., Liu, Y.: Stability of the $\mu$-Camassa-Holm peakons. J. Nonlinear Sci. 23, 97-112 (2013)

11. Bressan, A., Constantin, A.: Global conservative solutions of the Camassa-Holm equation. Arch. Ration. Mech. Anal. 183(2), 215-239 (2007)

12. Bressan, A., Constantin, A.: Global dissipative solutions of the Camassa-Holm equation. Anal. Appl. 5(1), 1-27 (2007)

13. Holden, H., Raynaud, X.: Periodic conservative solutions of the Camassa-Holm equation. Ann. Inst. Fourier (Grenoble) 58(3), 945-988 (2008)

14. Holden, H., Raynaud, X.: Dissipative solutions for the Camassa-Holm equation. Discrete Contin. Dyn. Syst. 24(4), 1047-1112 (2009)

15. Lenells, J.: Weak geodesic flow and global solutions of the Hunter-Saxton equation. Discrete Contin. Dyn. Syst. 18(4), 643-656 (2007)

16. Lee, J.M.: Geometric approach on the global conservative solutions of the Camassa-Holm equation. J. Geom. Phys. $142,137-150(2019)$

17. McKean, H.: Fredholm determinants and the Camassa-Holm hierarchy. Commun. Pure Appl. Math. 56(5), 638-680 (2003)

18. Tiğlay, F.: Conservative weak solutions of the periodic Cauchy problem for $\mu$ HS equation. J. Math. Phys. 56, Article ID 021504 (2015)

19. Hartman, P.: Ordinary Differential Equations. Birkhäuser, Basel (1982)

20. Gallouët, T., Vialard, F.X.: The Camassa-Holm equation as an incompressible Euler equation: a geometric point of view. J. Differ. Equ. 264(7), 4199-4234 (2018) 